\title{
Adaptive Training for Voice Conversion Based on Eigenvoices
}

\author{
Yamato OHTANI $^{\dagger \mathrm{a})}$, Student Member, Tomoki TODA ${ }^{\dagger \mathrm{b})}$, Hiroshi SARUWATARI ${ }^{\dagger \mathrm{c})}$, Members, , \\ and Kiyohiro SHIKANO ${ }^{\dagger \mathrm{d})}$, Fellow
}

\begin{abstract}
SUMMARY In this paper, we describe a novel model training method for one-to-many eigenvoice conversion (EVC). One-to-many EVC is a technique for converting a specific source speaker's voice into an arbitrary target speaker's voice. An eigenvoice Gaussian mixture model (EVGMM) is trained in advance using multiple parallel data sets consisting of utterance-pairs of the source speaker and many pre-stored target speakers. The EV-GMM can be adapted to new target speakers using only a few of their arbitrary utterances by estimating a small number of adaptive parameters. In the adaptation process, several parameters of the EV-GMM to be fixed for different target speakers strongly affect the conversion performance of the adapted model. In order to improve the conversion performance in one-to-many EVC, we propose an adaptive training method of the EV-GMM. In the proposed training method, both the fixed parameters and the adaptive parameters are optimized by maximizing a total likelihood function of the EV-GMMs adapted to individual pre-stored target speakers. We conducted objective and subjective evaluations to demonstrate the effectiveness of the proposed training method. The experimental results show that the proposed adaptive training yields significant quality improvements in the converted speech.

key words: speech synthesis, voice conversion, Gaussian mixture model, eigenvoice, adaptive training
\end{abstract}

\section{Introduction}

Voice conversion (VC) is a technique for modifying nonlinguistic information such as voice characteristics while not changing linguistic information. One of the typical VC applications is speaker conversion [1], which is a technique for converting the voice of a given speaker, called the source speaker, so that it sounds like that of another speaker, called the target speaker. This application can be extended to crosslanguage speaker conversion [2], [3], which is a technique for making it possible to generate various languages with our own voices even if we cannot speak those languages.

Recently, a conversion method based on the Gaussian mixture model (GMM) [4] has been studied widely. In this method, a GMM of joint probability density of source and target acoustic features is trained in advance using a parallel data set consisting of utterance-pairs of the source and target speakers. The trained GMM enables us to convert the source

\footnotetext{
Manuscript received October 5, 2009.

Manuscript revised January 15, 2010.

${ }^{\dagger}$ The authors are with the Graduate School of Information Science, Nara Institute of Science and Technology, Ikoma-shi, 6300192 Japan.

a) E-mail: yamato-o@is.naist.jp

b)E-mail: tomoki@is.naist.jp

c)E-mail: sawatari@is.naist.jp

d) E-mail: shikano@is.naist.jp

DOI: 10.1587/transinf.E93.D.1589
}

acoustic features of any utterance into the target acoustic features in a probabilistic manner [5]. Although this method works reasonably well, this training framework using the parallel data set causes many limitations of VC applications due to its lower flexibility.

In order to relax the use of the parallel data set, several approaches to flexibly building a GMM for a desired speaker-pair by effectively using another GMM for a different speaker-pair have been studied, e.g., [6] and [7]. As one of these approaches, we have proposed eigenvoice conversion (EVC) [8] inspired by an eigenvoice technique [9] that was originally proposed as a model adaptation technique in speech recognition. One of the specific features of EVC is to use more informative prior knowledge for building a new GMM, which is extracted from many other different speakers. As one of the main frameworks in EVC [10], we have so far proposed one-to-many EVC that allows the conversion from a specific source speaker's voice into arbitrary target speakers' voices. An eigenvoice GMM (EV-GMM) is trained in advance using multiple parallel data sets consisting of the source speaker and many pre-stored target speakers. The EV-GMM is capable of being flexibly adapted to a new target speaker using only a few arbitrary utterances of the target speaker. In this adaptation process, a few adaptive parameters are estimated in a completely text-independent manner. Moreover, the EV-GMM also allows us to control voice quality of the converted speech by manipulating those adaptive parameters manually.

The EV-GMM consists of tied-parameters that are fixed over different target speakers and adaptive parameters that are determined according to each of the individual target speakers. In the conventional training method of the EV-GMM, we build the EV-GMM using parameters from a target-speaker-independent GMM. These parameters are strongly affected by acoustic variations among many pre-stored target speakers. They usually cause significant degradation of the conversion performance of the adapted EV-GMM. This is a well-known issue often observed in speech recognition and it is effectively alleviated by using a pseudo-normalized speaker model, called the canonical model, rather than a speaker-independent model as an initial model for speaker adaptation. It has been reported that adaptive training such as speaker adaptive training (SAT) [11] or cluster adaptive training (CAT) [12] is a very effective paradigm for training the canonical model. Inspired by these studies, we propose an adaptive training method of the EV- 
GMM in one-to-many EVC. Moreover, we propose methods for alleviating the local optimum problem often caused in the proposed adaptive training when the number of adaptive parameters is set to be low. The experimental results of objective and subjective evaluations demonstrate that the proposed adaptive training yields significant quality improvements in converted speech.

The paper is organized as follows. In Sect. 2, we describe a basic algorithm of EVC. In Sect. 3, our proposed adaptive training method for the EV-GMM is described. In Sect. 4, we describe experimental evaluations. Finally, we summarize this paper in Sect. 5 .

\section{Eigenvoice Conversion}

\subsection{Eigenvoice Gaussian Mixture Model (EV-GMM)}

We use $2 D$-dimensional acoustic features, the source speaker's feature at the $t^{\text {th }}$ frame $\boldsymbol{X}_{t}=\left[\boldsymbol{x}_{t}^{\top}, \Delta \boldsymbol{x}_{t}^{\top}\right]^{\top}$ and the $s^{\text {th }}$ target speaker's feature at the $t^{\text {th }}$ frame $\boldsymbol{Y}_{t}^{(s)}=$ $\left[\boldsymbol{y}_{t}^{(s)^{\top}}, \Delta \boldsymbol{y}_{t}^{(s)^{\top}}\right]^{\top}$, consisting of $D$-dimensional static and dynamic features, where $T$ denotes transposition of the vector. Joint probability density of $\boldsymbol{Z}_{t}^{(s)}=\left[\boldsymbol{X}_{t}^{\top}, \boldsymbol{Y}_{t}^{(s)^{\top}}\right]^{\top}$ consisting of time-aligned source and target features determined by DTW is modeled with EV-GMM as follows:

$$
\begin{gathered}
P\left(\boldsymbol{Z}_{t}^{(s)} \mid \lambda^{(E V)}, \boldsymbol{w}^{(s)}\right) \\
=\sum_{m=1}^{M} \alpha_{m} \mathcal{N}\left(\boldsymbol{Z}_{t}^{(s)} ; \boldsymbol{\mu}_{m}^{(Z)}\left(\boldsymbol{w}^{(s)}\right), \boldsymbol{\Sigma}_{m}^{(Z Z)}\right), \\
\boldsymbol{\mu}_{m}^{(Z)}\left(\boldsymbol{w}^{(s)}\right)=\left[\begin{array}{c}
\boldsymbol{\mu}_{m}^{(X)} \\
\boldsymbol{B}_{m} \boldsymbol{w}^{(s)}+\boldsymbol{b}_{m}^{(0)}
\end{array}\right], \\
\boldsymbol{\Sigma}_{m}^{(Z Z)}=\left[\begin{array}{cc}
\boldsymbol{\Sigma}_{m}^{(X X)} & \boldsymbol{\Sigma}_{m}^{(X Y)} \\
\boldsymbol{\Sigma}_{m}^{(Y X)} & \boldsymbol{\Sigma}_{m}^{(Y Y)}
\end{array}\right],
\end{gathered}
$$

where $\mathcal{N}(x ; \mu, \boldsymbol{\Sigma})$ denotes Gaussian distribution with mean vector $\mu$ and covariance matrix $\boldsymbol{\Sigma}$, and $M$ represents the number of mixture components. In the EV-GMM, a target mean vector is modeled as a linear combination with the bias vector $\boldsymbol{b}_{m}^{(0)}$, representative vectors $\boldsymbol{B}_{m}=$ $\left[\boldsymbol{b}_{m}^{(1)}, \boldsymbol{b}_{m}^{(2)}, \cdots, \boldsymbol{b}_{m}^{(J)}\right]$ and the weight vector $\boldsymbol{w}^{(s)}$. Voice characteristics of various target speakers are effectively modeled by setting $\boldsymbol{w}^{(s)}$ to appropriate values. The other parameters $\lambda^{(E V)}$ such as mixture component weights, source mean vectors, bias vectors, representative vectors and covariance matrices are tied for every target speaker.

\subsection{Training of EV-GMM Based on Principal Component Analysis}

First, a target-speaker-independent GMM (TI-GMM) $\lambda^{(0)}$ is trained with multiple parallel data sets consisting of utterance-pairs of the source speaker and multiple prestored target speakers. Then, using only the parallel data set for the $s^{\text {th }}$ pre-stored target speaker, the $s^{\text {th }}$ target-speaker-dependent GMM (TD-GMM) $\lambda^{(s)}$ is trained by only updating target mean vectors of $\lambda^{(0)}$. After training the TD-GMMs for all pre-stored target speakers, a $2 D M$-dimensional supervector $\boldsymbol{S} \boldsymbol{V}^{(s)}=$ $\left[\boldsymbol{\mu}_{1}^{(Y)}(s), \boldsymbol{\mu}_{2}^{(Y)}(s), \cdots, \boldsymbol{\mu}_{M}^{(Y)^{\top}}(s)\right]^{\top}$ is constructed for each prestored target speaker by concatenating the updated target mean vectors $\left\{\boldsymbol{\mu}_{1}^{(Y)}(s), \boldsymbol{\mu}_{2}^{(Y)}(s), \cdots, \boldsymbol{\mu}_{M}^{(Y)}(s)\right\}$ of $\lambda^{(s)}$. Finally, bias vector $\boldsymbol{b}_{m}^{(0)}$ and representative vectors $\boldsymbol{B}_{m}$ are extracted by performing principal component analysis (PCA) for the supervectors for all pre-stored target speakers $\left\{\boldsymbol{S} \boldsymbol{V}^{(1)}, \boldsymbol{S} \boldsymbol{V}^{(2)}, \cdots, \boldsymbol{S} \boldsymbol{V}^{(S)}\right\}$, where $S$ denotes the number of pre-stored target speakers. Consequently, each supervector is approximated as follows:

$$
\begin{aligned}
\boldsymbol{S} \boldsymbol{V}^{(s)} \simeq & {\left[\boldsymbol{B}_{1}^{\top}, \boldsymbol{B}_{2}^{\top}, \cdots, \boldsymbol{B}_{M}^{\top}\right]^{\top} \boldsymbol{w}^{(s)} } \\
& +\left[\boldsymbol{b}_{1}^{(0)^{\top}}, \boldsymbol{b}_{1}^{(0)^{\top}}, \cdots, \boldsymbol{b}_{M}^{(0)^{\top}}\right]^{\top}, \\
\boldsymbol{b}_{m}^{(0)}= & \frac{1}{S} \sum_{s=1}^{S} \boldsymbol{\mu}_{m}^{(Y)}(s),
\end{aligned}
$$

where $\boldsymbol{w}^{(s)}$ is $J(<S \ll 2 D M)$ principle components for the $s^{\text {th }}$ target speaker.

\subsection{Unsupervised Adaptation of EV-GMM}

The EV-GMM is adapted for arbitrary target speakers by estimating the optimum weight vector for their given speech samples without any linguistic information. We apply maximum likelihood eigen-decomposition [9] to the weight estimation. In one-to-many EVC, the weight vector $\boldsymbol{w}$ is estimated so that likelihood of the marginal distribution for a time sequence of the given target features $\boldsymbol{Y}^{(t a r)}$ is maximized [8] as follows:

$$
\begin{aligned}
\hat{\boldsymbol{w}} & =\arg \max _{\boldsymbol{w}} \int P\left(\boldsymbol{X}, \boldsymbol{Y}^{(\text {tar })} \mid \lambda^{(E V)}, \boldsymbol{w}\right) d \boldsymbol{X} \\
& =\arg \max _{\boldsymbol{w}} P\left(\boldsymbol{Y}^{(t a r)} \mid \lambda^{(E V)}, \boldsymbol{w}\right) .
\end{aligned}
$$

The maximum likelihood (ML) estimate of the weight vector for a target speaker $\hat{\boldsymbol{w}}$ is determined as follows:

$$
\begin{aligned}
\hat{\boldsymbol{w}}= & \left\{\sum_{m=1}^{M} \bar{\gamma}_{m}^{(\text {tar })} \boldsymbol{B}_{m}^{\top} \boldsymbol{\Sigma}_{m}^{(Y Y)^{-1}} \boldsymbol{B}_{m}\right\}^{-1} \\
& \times \sum_{m=1}^{M} \boldsymbol{B}_{m}^{\top} \boldsymbol{\Sigma}_{m}^{(Y Y)^{-1}} \overline{\boldsymbol{Y}}_{m}^{(t a r)}
\end{aligned}
$$

where

$$
\begin{aligned}
& \bar{\gamma}_{m}^{(t a r)}=\sum_{t=1}^{T} P\left(m \mid \boldsymbol{Y}_{t}^{(t a r)}, \lambda^{(E V)}, \boldsymbol{w}\right), \\
& \overline{\boldsymbol{Y}}_{t}^{(t a r)}=\sum_{t=1}^{T} P\left(m \mid \boldsymbol{Y}_{t}^{(t a r)}, \lambda^{(E V)}, \boldsymbol{w}\right)\left(\boldsymbol{Y}_{t}^{(t a r)}-\boldsymbol{b}_{m}^{(0)}\right) .
\end{aligned}
$$


Note that this estimation process is a completely unsupervised adaptation process because parallel data is not necessary and there is no linguistic limitation in the target adaptation data.

\subsection{Conversion with Adapted EV-GMM}

We use the conversion method based on ML estimation considering dynamic features [5]. Let a time sequence of the source features and that of the target features be $\boldsymbol{X}=\left[\boldsymbol{X}_{1}^{\top}, \boldsymbol{X}_{2}^{\top}, \cdots, \boldsymbol{X}_{T}^{\top}\right]^{\top}$ and $\boldsymbol{Y}=\left[\boldsymbol{Y}_{1}^{\top}, \boldsymbol{Y}_{2}^{\top}, \cdots, \boldsymbol{Y}_{T}^{\top}\right]^{\top}$, respectively. Converted static feature vectors $\hat{\boldsymbol{y}}=$ $\left[\hat{\boldsymbol{y}}_{1}^{\top}, \hat{\boldsymbol{y}}_{2}^{\top}, \cdots, \hat{\boldsymbol{y}}_{T}^{\top}\right]^{\top}$ are obtained as follows:

$$
\hat{\boldsymbol{y}}=\arg \max _{\boldsymbol{y}} \sum_{\text {all } \boldsymbol{m}} P\left(\boldsymbol{m} \mid \boldsymbol{X}, \lambda^{(E V)}\right) P\left(\boldsymbol{Y} \mid \boldsymbol{X}, \boldsymbol{m}, \lambda^{(E V)}, \hat{\boldsymbol{w}}\right) .
$$

This likelihood function is approximated with the suboptimum mixture component sequence $\hat{\boldsymbol{m}}=\left[\hat{m}_{1}, \hat{m}_{2}, \cdots, \hat{m}_{T}\right]$, which is determined by

$$
\hat{\boldsymbol{m}}=\arg \max _{\boldsymbol{m}} P\left(\boldsymbol{m} \mid \boldsymbol{X}, \lambda^{(E V)}\right) .
$$

Then, we determined the converted static feature sequence $\hat{\boldsymbol{y}}$ as follows:

$$
\begin{aligned}
& \hat{\boldsymbol{y}}=\arg \max _{\boldsymbol{y}} P\left(\boldsymbol{Y} \mid \boldsymbol{X}, \hat{\boldsymbol{m}}, \lambda^{(E V)}, \hat{\boldsymbol{w}}\right), \\
& \text { subject to } \boldsymbol{Y}=\boldsymbol{W} \boldsymbol{y},
\end{aligned}
$$

where $\boldsymbol{W}$ denotes the matrix to extend the static feature sequence to the static and dynamic feature sequence, and $P\left(\boldsymbol{Y} \mid \boldsymbol{X}, \hat{\boldsymbol{m}}, \lambda^{(E V)}, \hat{\boldsymbol{w}}\right)$ is written as

$$
\begin{aligned}
& P\left(\boldsymbol{Y} \mid \boldsymbol{X}, \hat{\boldsymbol{m}}, \lambda^{(E V)}, \hat{\boldsymbol{w}}\right)=\prod_{t=1}^{T} \mathcal{N}\left(\boldsymbol{Y}_{t} ; \boldsymbol{E}_{\hat{m}_{t}, t}^{(Y)}, \boldsymbol{D}_{\hat{m}_{t}}^{(Y)}\right),
\end{aligned}
$$

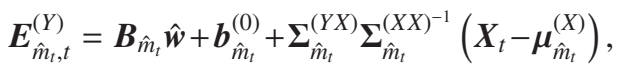

$$
\begin{aligned}
& \boldsymbol{D}_{\hat{m}_{t}}^{(Y)}=\boldsymbol{\Sigma}_{\hat{m}_{t}}^{(Y Y)}-\boldsymbol{\Sigma}_{\hat{m}_{t}}^{(Y X)} \boldsymbol{\Sigma}_{\hat{m}_{t}}^{(X X)^{-1}} \boldsymbol{\Sigma}_{\hat{m}_{t}}^{(X Y)} .
\end{aligned}
$$

The converted static feature sequence $\hat{\boldsymbol{y}}$ is given by

$$
\hat{\boldsymbol{y}}=\left(\boldsymbol{W}^{\top} \boldsymbol{D}_{\hat{\boldsymbol{m}}}^{(Y)^{-1}} \boldsymbol{W}\right)^{-1} \boldsymbol{W}^{\top} \boldsymbol{D}_{\hat{\boldsymbol{m}}}^{(Y)^{-1}} \boldsymbol{E}_{\hat{\boldsymbol{m}}}^{(Y)},
$$

where,

$$
\begin{aligned}
& \boldsymbol{E}_{\hat{\boldsymbol{m}}}^{(Y)}=\left[\boldsymbol{E}_{m_{1}, 1}^{(Y)}, \boldsymbol{E}_{m_{2}, 2}^{(Y)}, \cdots, \boldsymbol{E}_{m_{T}, T}^{(Y)}\right], \\
& \boldsymbol{D}_{\hat{\boldsymbol{m}}}^{(Y)^{-1}}=\operatorname{diag}\left[\boldsymbol{D}_{m_{1}}^{(Y)^{-1}}, \boldsymbol{D}_{m_{2}}^{(Y)^{-1}}, \cdots, \boldsymbol{D}_{m_{T}}^{(Y)^{-1}}\right] .
\end{aligned}
$$

Moreover, the converted speech quality is dramatically improved by further considering global variance [5], which is defined as the variance of the target static features calculated over a time sequence.

\subsection{Problem of PCA-Based EV-GMM}

The tied-parameters of the PCA-based EV-GMM are from

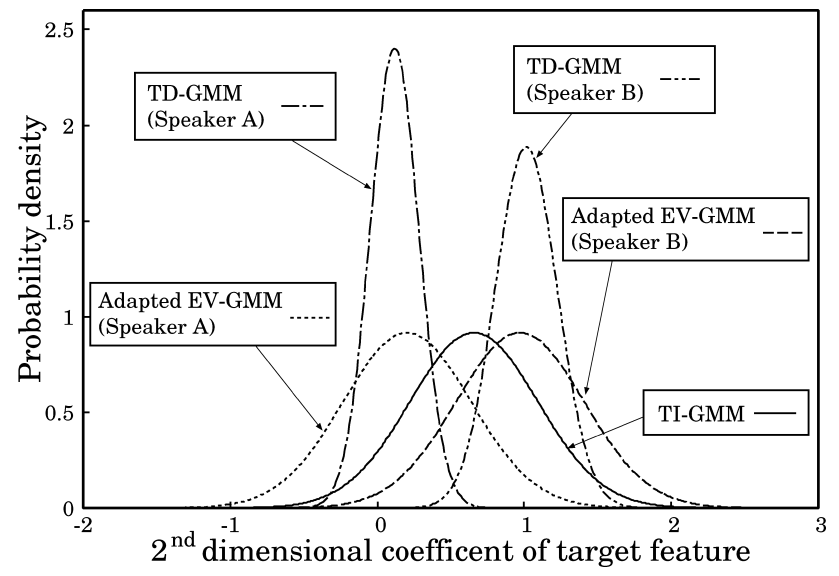

Fig. 1 Marginal distributions for the $2^{\text {nd }}$ dimensional coefficient of target features of TI-GMM, TD-GMMs and adapted EV-GMMs.

the TI-GMM modeling joint probability density of acoustic features of the source speaker and all pre-stored target speakers as mentioned. They capture not only intraspeaker acoustic variations but also inter-speaker acoustic variations of pre-stored target speakers. To demonstrate the impact of these tied-parameters on the EV-GMM adaptation, Fig. 1 shows marginal distributions of the TI-GMM, two TD-GMMs and two adapted EV-GMMs on the $2^{\text {nd }}$ dimensional coefficient of target features. We can see that variance values of the adapted EV-GMMs are much larger than those of the TD-GMMs although their mean values are close to those of the TD-GMMs. These mismatches in modeling probability density would cause performance degradation of the adapted EV-GMM.

\section{Adaptive Training for EV-GMM}

In order to alleviate the mismatch issues observed in the PCA-based EV-GMM, we propose an adaptive training method for the one-to-many EV-GMM. A canonical EVGMM for the EV-GMM adaptation is trained in the adaptive training paradigm so that the performance of the adapted EV-GMMs is improved.

\subsection{Training Algorithm}

The canonical EV-GMM is trained by maximizing the total likelihood of the adapted EV-GMMs for individual pre-stored target speakers with respect to both canonical EV-GMM parameters (i.e., the tied-parameters of the EVGMM $\lambda^{(E V)}$ ) and target-speaker adaptive parameters (i.e., the weight vector $\boldsymbol{w}^{(s)}$ for each pre-stored target speaker) as follows:

$$
\left\{\hat{\lambda}^{(E V)}, \hat{\boldsymbol{w}}_{1}^{S}\right\}=\arg \max _{\lambda^{(E V)}, \boldsymbol{w}_{1}^{S}} \prod_{s=1}^{S} \prod_{t=1}^{T_{s}} P\left(\boldsymbol{Z}_{t}^{(s)} \mid \lambda^{(E V)}, \boldsymbol{w}^{(s)}\right),
$$

where $\boldsymbol{w}_{1}^{S}$ is a set of weight vectors for individual pre-stored target speakers $\left\{\boldsymbol{w}^{(1)}, \boldsymbol{w}^{(2)}, \cdots, \boldsymbol{w}^{(S)}\right\}$. The training process 
is performed with EM algorithm by maximizing the following auxiliary function,

$$
\begin{aligned}
& Q\left(\left\{\lambda^{(E V)}, \boldsymbol{w}_{1}^{S}\right\},\left\{\hat{\lambda}^{(E V)}, \hat{\boldsymbol{w}}_{1}^{S}\right\}\right) \\
= & \sum_{s=1}^{S} \sum_{t=1}^{T_{s}} \sum_{m=1}^{M} \gamma_{m, t}^{(s)} \log P\left(\boldsymbol{Z}_{t}^{(s)}, m \mid \hat{\lambda}^{(E V)}, \hat{\boldsymbol{w}}^{(s)}\right),
\end{aligned}
$$

where the first variable in the auxiliary function (i.e., $\left\{\lambda^{(E V)}, \boldsymbol{w}_{1}^{S}\right\}$ in Eq. (21)) is a parameter set used in E-step for calculating the following posterior probability

$$
\gamma_{m, t}^{(s)}=P\left(m \mid Z_{t}^{(s)}, \lambda^{(E V)}, \boldsymbol{w}^{(s)}\right),
$$

and the other variable in it (i.e., $\left\{\hat{\lambda}^{(E V)}, \hat{\boldsymbol{w}}_{1}^{S}\right\}$ in Eq. (21)) is a parameter set to be updated in M-step, which is used for calculating the $\log$-scaled likelihood $\log P\left(\boldsymbol{Z}_{t}^{(s)}, m \mid \hat{\lambda}^{(E V)}, \hat{\boldsymbol{w}}^{(s)}\right)$. It is difficult to update all parameters simultaneously because some of them depend on each other. Therefore, each parameter of EV-GMM is updated as follows:

$$
\begin{aligned}
& Q\left(\left\{\lambda^{(E V)}, \boldsymbol{w}_{1}^{S}\right\},\left\{\lambda^{(E V)}, \boldsymbol{w}_{1}^{S}\right\}\right) \\
& \leq Q\left(\left\{\lambda^{(E V)}, \boldsymbol{w}_{1}^{S}\right\},\left\{\alpha_{m}, \boldsymbol{\Sigma}_{m}^{(Z Z)}, \boldsymbol{B}_{m}, \boldsymbol{b}_{m}^{(0)}, \boldsymbol{\mu}_{m}^{(X)}, \hat{\boldsymbol{w}}_{1}^{S}\right\}\right) \\
& \leq Q\left(\left\{\lambda^{(E V)}, \boldsymbol{w}_{1}^{S}\right\},\left\{\alpha_{m}, \boldsymbol{\Sigma}_{m}^{(Z Z)}, \hat{\boldsymbol{B}}_{m}, \hat{\boldsymbol{b}}_{m}^{(0)}, \hat{\boldsymbol{\mu}}_{m}^{(X)}, \hat{\boldsymbol{w}}_{1}^{S}\right\}\right) \\
& \leq Q\left(\left\{\lambda^{(E V)}, \boldsymbol{w}_{1}^{S}\right\},\left\{\hat{\alpha}_{m}, \hat{\boldsymbol{\Sigma}}_{m}^{(Z Z)}, \hat{\boldsymbol{B}}_{m}, \hat{\boldsymbol{b}}_{m}^{(0)}, \hat{\boldsymbol{\mu}}_{m}^{(X)}, \hat{\boldsymbol{w}}_{1}^{S}\right\}\right) .
\end{aligned}
$$

It is sufficient to ensure these updates to satisfy

$$
\begin{aligned}
& \prod_{s=1}^{S} \prod_{t=1}^{T_{s}} P\left(\boldsymbol{Z}_{t}^{(s)}, m \mid \lambda^{(E V)}, \boldsymbol{w}^{(s)}\right) \\
& \leq \prod_{s=1}^{S} \prod_{t=1}^{T_{s}} P\left(\boldsymbol{Z}_{t}^{(s)}, m \mid\left\{\alpha_{m}, \boldsymbol{\Sigma}_{m}^{(Z Z)}, \boldsymbol{B}_{m}, \boldsymbol{b}_{m}^{(0)}, \boldsymbol{\mu}_{m}^{(X)}\right\}, \hat{\boldsymbol{w}}^{(s)}\right) \\
& \leq \prod_{s=1}^{S} \prod_{t=1}^{T_{s}} P\left(\boldsymbol{Z}_{t}^{(s)}, m \mid\left\{\alpha_{m}, \boldsymbol{\Sigma}_{m}^{(Z Z)}, \hat{\boldsymbol{B}}_{m}, \hat{\boldsymbol{b}}_{m}^{(0)}, \hat{\boldsymbol{\mu}}_{m}^{(X)}\right\}, \hat{\boldsymbol{w}}^{(s)}\right) \\
& \leq \prod_{s=1}^{S} \prod_{t=1}^{T_{s}} P\left(\boldsymbol{Z}_{t}^{(s)}, m \mid\left\{\hat{\alpha}_{m}, \hat{\boldsymbol{\Sigma}}_{m}^{(Z Z)}, \hat{\boldsymbol{B}}_{m}, \hat{\boldsymbol{b}}_{m}^{(0)}, \hat{\boldsymbol{\mu}}_{m}^{(X)}\right\}, \hat{\boldsymbol{w}}^{(s)}\right) .
\end{aligned}
$$

These update processes are iteratively performed in each M-step for improving parameter estimation accuracy. ML estimate of the weight vector for the $s^{\text {th }}$ pre-stored target speaker is written as

$$
\begin{aligned}
\hat{\boldsymbol{w}}^{(s)}=\left(\sum_{m=1}^{M} \bar{\gamma}_{m}^{(s)} \boldsymbol{B}_{m}^{\top} \boldsymbol{P}_{m}^{(Y Y)} \boldsymbol{B}_{m}\right)^{-1} \\
\times\left[\sum _ { m = 1 } ^ { M } \left\{\boldsymbol{B}_{m}^{\top} \boldsymbol{P}_{m}^{(Y X)}\left(\overline{\boldsymbol{X}}_{m}^{(s)}-\bar{\gamma}_{m}^{(s)} \boldsymbol{\mu}_{m}^{(X)}\right)\right.\right. \\
\left.\left.+\boldsymbol{B}_{m}^{\top} \boldsymbol{P}_{m}^{(Y Y)}\left(\overline{\boldsymbol{Y}}_{m}^{(s)}-\bar{\gamma}_{m}^{(s)} \boldsymbol{b}_{m}^{(0)}\right)\right\}\right],
\end{aligned}
$$

where

$$
\begin{aligned}
& \bar{\gamma}_{m}^{(s)}=\sum_{t=1}^{T_{s}} P\left(m \mid \boldsymbol{Z}_{t}^{(s)}, \lambda^{(E V)}, \boldsymbol{w}^{(s)}\right), \\
& \overline{\boldsymbol{X}}_{m}^{(s)}=\sum_{t=1}^{T s} P\left(m \mid \boldsymbol{Z}_{t}^{(s)}, \lambda^{(E V)}, \boldsymbol{w}^{(s)}\right) \boldsymbol{X}_{t}, \\
& \overline{\boldsymbol{Y}}_{m}^{(s)}=\sum_{t=1}^{T_{s}} P\left(m \mid \boldsymbol{Z}_{t}^{(s)}, \lambda^{(E V)}, \boldsymbol{w}^{(s)}\right) \boldsymbol{Y}_{t}^{(s)}, \\
& \boldsymbol{\Sigma}_{m}^{(Z Z)^{-1}}=\left[\begin{array}{ll}
\boldsymbol{P}_{m}^{(X X)} & \boldsymbol{P}_{m}^{(X Y)} \\
\boldsymbol{P}_{m}^{(Y X)} & \boldsymbol{P}_{m}^{(Y Y)}
\end{array}\right] .
\end{aligned}
$$

ML estimates of the tied-parameters for mean vectors are written as

$$
\hat{\boldsymbol{v}}_{m}=\left(\sum_{s=1}^{S} \bar{\gamma}_{m}^{(s)} \hat{\boldsymbol{W}}_{s}^{\top} \boldsymbol{\Sigma}_{m}^{(Z Z)^{-1}} \hat{\boldsymbol{W}}_{s}\right)^{-1}\left(\sum_{s=1}^{S} \hat{\boldsymbol{W}}_{s}^{\top} \boldsymbol{\Sigma}_{m}^{(Z Z)^{-1}} \overline{\boldsymbol{Z}}_{m}^{(s)}\right),
$$

where

$$
\begin{aligned}
\overline{\boldsymbol{Z}}_{m}^{(s)} & =\left[\begin{array}{c}
\overline{\boldsymbol{X}}_{m}^{(s)} \\
\overline{\boldsymbol{Y}}_{m}^{(s)}
\end{array}\right], \\
\hat{\boldsymbol{v}}_{m} & =\left[\begin{array}{lllll}
\hat{\boldsymbol{\mu}}_{m}^{(X)^{\top}}, \hat{\boldsymbol{b}}_{m}^{(0)^{\top}}, \hat{\boldsymbol{b}}_{m}^{(1)^{\top}}, \cdots, & \cdots, \hat{\boldsymbol{b}}_{m}^{(J)^{\top}}
\end{array}\right]^{\top}, \\
\hat{\boldsymbol{W}}_{s} & =\left[\begin{array}{cccccc}
\boldsymbol{I} & \mathbf{0} & \mathbf{0} & \mathbf{0} & \cdots & \mathbf{0} \\
\mathbf{0} & \boldsymbol{I} & \hat{w}_{1}^{(s)} \boldsymbol{I} & \hat{w}_{2}^{(s)} \boldsymbol{I} & \cdots & \hat{w}_{J}^{(s)} \boldsymbol{I}
\end{array}\right],
\end{aligned}
$$

and the matrix $\boldsymbol{I}$ is a $D \times D$ identity matrix. Then, mixture component weights and covariance matrices are determined as follows:

$$
\begin{gathered}
\hat{\alpha}_{m}=\frac{\sum_{s=1}^{S} \bar{\gamma}_{m}^{(s)}}{\sum_{m=1}^{M} \sum_{s=1}^{S} \bar{\gamma}_{m}^{(s)}}, \\
\hat{\boldsymbol{\Sigma}}_{m}^{(Z Z)}=\frac{1}{\sum_{s=1}^{S} \bar{\gamma}_{m}^{(s)}} \sum_{s=1}^{S}\left\{\overline{\boldsymbol{V}}_{m}^{(s)}+\bar{\gamma}_{m}^{(s)} \hat{\boldsymbol{\mu}}_{m}^{(s)} \hat{\boldsymbol{\mu}}_{m}^{(s)^{\top}}\right. \\
\left.\quad-\left(\hat{\boldsymbol{\mu}}_{m}^{(s)} \overline{\boldsymbol{Z}}_{m}^{(s)^{\top}}+\overline{\boldsymbol{Z}}_{m}^{(s)} \hat{\boldsymbol{\mu}}_{m}^{(s)^{\top}}\right)\right\},
\end{gathered}
$$

where

$$
\begin{aligned}
& \overline{\boldsymbol{V}}_{m}^{(s)}=\sum_{t=1}^{T_{s}} P\left(m \mid \boldsymbol{Z}_{t}^{(s)}, \lambda^{(E V)}, \boldsymbol{w}^{(s)}\right) \boldsymbol{Z}_{t}^{(s)} \boldsymbol{Z}_{t}^{(s)^{\top}}, \\
& \hat{\boldsymbol{\mu}}_{m}^{(s)}=\hat{\boldsymbol{W}}_{s} \hat{\boldsymbol{v}}_{m}=\left[\begin{array}{c}
\hat{\boldsymbol{\mu}}_{m}^{(X)} \\
\hat{\boldsymbol{B}}_{m} \hat{\boldsymbol{w}}^{(s)}+\hat{\boldsymbol{b}}_{m}^{(0)}
\end{array}\right] .
\end{aligned}
$$

In Eq. (30), we need to calculate $\{D(J+2)\} \times$ $\{D(J+2)\}$-sized inverse matrix. If using diagonal covariance matrices for $\boldsymbol{\Sigma}^{(X X)}, \boldsymbol{\Sigma}^{(X Y)}, \boldsymbol{\Sigma}^{(Y X)}$ and $\boldsymbol{\Sigma}^{(Y Y)}$, the computational cost is significantly reduced by calculating the ML estimate of $\boldsymbol{v}_{m}$ dimension-by-dimension as follows: 


$$
\hat{\boldsymbol{v}}_{m}^{(d)}=\left(\sum_{s=1}^{S} \bar{\gamma}_{m}^{(s)} \hat{\boldsymbol{W}}_{s}^{\prime \top} \boldsymbol{P}_{m, d}^{(Z Z)} \hat{\boldsymbol{W}}_{s}^{\prime}\right)^{-1}\left(\sum_{s=1}^{S} \hat{\boldsymbol{W}}_{s}^{{ }^{\top}} \boldsymbol{P}_{m, d}^{(Z Z)} \overline{\boldsymbol{Z}}_{m, d}^{(s)}\right),
$$

where

$$
\begin{aligned}
& \hat{\boldsymbol{v}}_{m}^{(d)}=\left[\hat{\mu}_{m, d}^{(X)}, \hat{b}_{m, d}^{(0)}, \hat{b}_{m, d}^{(1)}, \cdots, \hat{b}_{m, d}^{(J)}\right]^{\top}, \\
& \hat{\boldsymbol{W}}_{s}^{\prime}=\left[\begin{array}{cccccc}
1 & 0 & 0 & 0 & \cdots & 0 \\
0 & 1 & \hat{w}_{1}^{(s)} & \hat{w}_{2}^{(s)} & \cdots & \hat{w}_{J}^{(s)}
\end{array}\right], \\
& \boldsymbol{P}_{m, d}^{(Z Z)}=\left[\begin{array}{ll}
p_{m, d}^{(X X)} & p_{m, d}^{(X Y)} \\
p_{m, d}^{(Y X)} & p_{m, d}^{(Y Y)}
\end{array}\right], \\
& \bar{Z}_{m, d}^{(s)}=\left[\bar{X}_{m, d}^{(s)}, \bar{Y}_{m, d}^{(s)}\right]^{\top} \text {. }
\end{aligned}
$$

The vector $\boldsymbol{v}_{m}^{(d)}$ consists of the $d^{\text {th }}$ dimensional components of $\boldsymbol{v}_{m}$, and $\mu_{m, d}^{(X)}$ and $b_{m, d}^{(j)}$ are the $d^{\text {th }}$ components of $\boldsymbol{\mu}_{m}^{(X)}$ and $\boldsymbol{b}_{m}^{(j)}$, respectively. In Eq. (41), $p_{m, d}^{(X X)}, p_{m, d}^{(X Y)}, p_{m, d}^{(Y X)}$ and $p_{m, d}^{(Y Y)}$ are the $d^{\text {th }}$ diagonal components of $\boldsymbol{P}_{m}^{(X X)}, \boldsymbol{P}_{m}^{(X Y)}, \boldsymbol{P}_{m}^{(Y X)}$ and $\boldsymbol{P}_{m}^{(Y Y)}$, respectively. In Eq. (42), $\bar{X}_{m, d}^{(s)}$ and $\bar{Y}_{m, d}^{(s)}$ are the $d^{\text {th }}$ components of $\overline{\boldsymbol{X}}_{m}^{(s)}$ and $\overline{\boldsymbol{Y}}_{m}^{(s)}$, respectively. We need to calculate $\{J+2\} \times\{J+2\}$-sized inverse matrices for each dimension.

\subsection{Alleviating Local Optimum Problem}

In the canonical EV-GMM training, it is essential to estimate the representative vectors spanning a sub-space, effectively modeling acoustic variations among all pre-stored target speakers in each mixture component. To achieve this, individual mixture-component occupancies calculated by Eq. (26) have to be enough large for every pre-stored target speaker. Figure 2 shows an example of the occupancies for one pre-stored target speaker, which are sorted in descending order. We can see that the occupancies calculated with the TI-GMM are more biased compared with those calculated with the TD-GMM for the same pre-stored target speaker. Because the TI-GMM needs to model wide varieties of acoustic features of all pre-stored target speakers, some mixture components model only acoustic features of a part of pre-stored target speakers. Consequently, a larger number of mixture components with lack of occupancies are observed in the TI-GMM compared with in the TD-GMM.

Figure 2 also shows the occupancies of the canonical EV-GMMs with 159 representative vectors and with only one representative vector, which are iteratively updated from the TI-GMM with the EM algorithm. Even if the occupancies are biased in the first E-step as observed in the TIGMM, the trained canonical EV-GMM with a large enough number of representative vectors has an occupancy distribution similar to that of the TD-GMM. However, if the number of representative vectors is very small, the occupancy distribution of the trained canonical EV-GMM remains biased. Namely, the initial occupancies strongly affect the final canonical EV-GMM unless the number of representative vectors is large enough to precisely model acoustic features

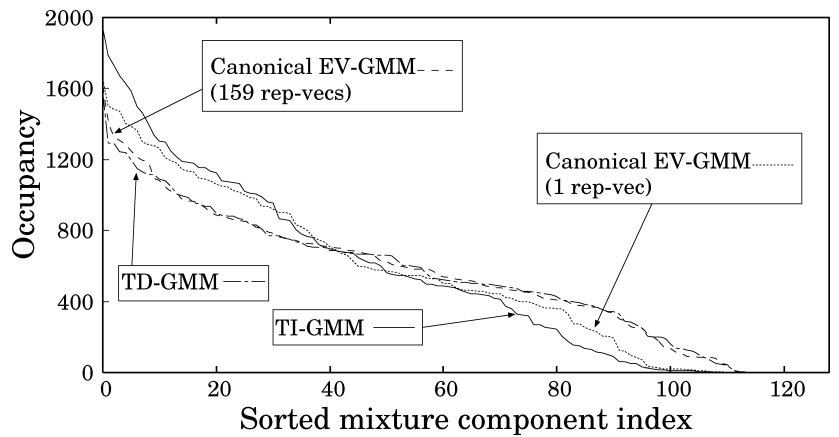

Fig. 2 Mixture-component occupancies for one pre-stored speaker using several models.

of individual pre-stored target speakers. This problem is critical because there are some situations where we prefer to train the EV-GMM with a small number of representative vectors, e.g., for keeping the computational cost of the adaptation process as low as possible or for reducing the model size as much as possible.

In order to alleviate this local optimum problem, we propose two approaches: 1) the TD-GMMs are used for calculating the occupancies in the first E-step; and 2) the deterministic annealing EM (DAEM) algorithm is used for the EV-GMM.

\subsubsection{First E-Step Approximation with Target-Speaker- Dependent Models}

Each of the TD-GMMs models the joint probability density for the source speaker and each pre-stored target speaker. Therefore, they generally yield more unbiased occupancies in each mixture component for every pre-stored speaker as shown in Fig. 2. The use of these occupancies for estimating the representative vectors is supposed to be helpful for alleviating the local optimum problem. Therefore, we propose an occupancy approximation method using the TDGMMs for calculating the occupancies only in the first Estep. Note that the correspondence of each mixture component between every TD-GMM and the PCA-based EVGMM is known because only target mean vectors are updated with parallel data in building each TD-GMM in order to preserve the correspondence of each mixture component in a phonemic space [10]. We update the PCA-based EV-GMM parameters in the first M-step based on the occupancies calculated with the TD-GMMs. Therefore, the first E-step and M-step are no longer regarded as the EM algorithm. In all steps that follow, we use the updated EV-GMM parameters for calculating the occupancies as in the EM algorithm.

\subsubsection{Deterministic Annealing EM (DAEM)}

In order to alleviate the local optimum problem, we apply the deterministic annealing EM (DAEM) algorithm [13] to the adaptive training of the EV-GMM. The DAEM algorithm reformulates a maximization process of a likelihood 
function as a minimization process of free energy. In adaptive training of the EV-GMM based on the DAEM algorithm, parameters are estimated as follows:

$$
\begin{aligned}
& \hat{\lambda}^{(E V)}, \hat{\boldsymbol{w}}_{1}^{S}=\arg \min _{\lambda^{(E V)}, \boldsymbol{w}_{1}^{S}} F_{\beta} \\
& F_{\beta}=-\frac{1}{\beta} \log \prod_{s=1}^{S} \prod_{t=1}^{T_{S}} \sum_{m=1}^{M} P\left(\boldsymbol{Z}_{t}^{(s)}, m \mid \lambda^{(E V)}, \boldsymbol{w}^{(s)}\right)^{\beta},
\end{aligned}
$$

where $1 / \beta$ is called the "temperature." The free energy given by Eq. (44) is minimized by maximizing the following auxiliary function,

$$
\begin{aligned}
& \left.Q_{\beta}\left(\left\{\lambda^{(E V)}, \boldsymbol{w}_{1}^{S}\right\},\left\{\hat{\lambda}^{(E V)}, \hat{\boldsymbol{w}}_{1}^{S}\right)\right\}\right) \\
= & \sum_{s=1}^{S} \sum_{t=1}^{T_{s}} \sum_{m=1}^{M} \gamma_{m, t, \beta}^{(s)} \log P\left(\boldsymbol{Z}_{t}^{(s)}, m \mid \hat{\lambda}^{(E V)}, \hat{\boldsymbol{w}}^{(s)}\right),
\end{aligned}
$$

where

$$
\gamma_{m, t, \beta}^{(s)}=\frac{P\left(\boldsymbol{Z}_{t}^{(s)}, m \mid \lambda^{(E V)}, \boldsymbol{w}^{(s)}\right)^{\beta}}{\sum_{m=1}^{M} P\left(\boldsymbol{Z}_{t}^{(s)}, m \mid \lambda^{(E V)}, \boldsymbol{w}^{(s)}\right)^{\beta}} .
$$

In the algorithm, we gradually decrease the temperature and deterministically optimize the function at each temperature. First $\beta$ is set to nearly zero so that the free energy function has a single global maximum, and the canonical model parameters and the adaptive parameters are iteratively updated by maximizing the auxiliary function. Then, we decrease $1 / \beta$ and update the parameters while fixing $\beta$. As $\beta$ gradually changes from nearly zero to 1 , the posterior distribution shown in Eq. (46) also gradually changes from a nearly uniform distribution to the original posterior distribution. Finally, we decrease $1 / \beta$ to 1 and update the parameters by maximizing the auxiliary function in Eq. (45), which is equivalent to the auxiliary function used in the EM algorithm.

\subsection{Discussion}

CAT [12] has been proposed as an adaptive training method for HMM-based speech recognition using the eigenvoice technique. There are some differences between CAT and the proposed method. The proposed adaptive training adapts only parameters on a part of the model space, i.e., the target feature space rather than the joint space. Consequently, the update formulas shown in Eq. (25) and (30) are different from those used in CAT. Moreover, the proposed method uses a GMM rather than HMMs. This makes an adaptation process much easier because the decoding process is inevitable if using HMMs. Consequently, the proposed method enables unsupervised adaptation in a linguistically independent manner.
Table 1 Number of pre-stored target speakers uttering each sub-set (A, $\mathrm{B}, \cdots$, or $\mathrm{G})$. Each sub-set consists of 50 phonetically balanced sentences.

\begin{tabular}{|l||c|c|c|c|c|c|c|c|}
\hline Sub-sets & A & B & C & D & E & F & G & Total \\
\hline $\begin{array}{l}\text { Number of male } \\
\text { speakers }\end{array}$ & 15 & 11 & 15 & 13 & 15 & 11 & 0 & 80 \\
\hline $\begin{array}{l}\text { Number of fe- } \\
\text { male speakers }\end{array}$ & 15 & 11 & 15 & 13 & 12 & 0 & 14 & 80 \\
\hline
\end{tabular}

Table 2 Relationship between number of representative vectors and contribution rate.

\begin{tabular}{|c|c|}
\hline $\begin{array}{c}\text { Number of } \\
\text { representative vectors }\end{array}$ & Contribution rate [\%] \\
\hline 1 & 22.49 \\
\hline 3 & 42.85 \\
\hline 8 & 61.95 \\
\hline 26 & 80.00 \\
\hline 159 & 100.0 \\
\hline
\end{tabular}

\section{Experimental Evaluations}

\subsection{Experimental Conditions}

We objectively and subjectively evaluated the conversion performance of the proposed canonical EV-GMM compared with that of the conventional PCA-based EV-GMM in oneto-many EVC. We used parallel data sets of a single source male speaker and 160 pre-stored target speakers consisting of 80 male and 80 female speakers for training the EV-GMM. These speakers were included in the Japanese Newspaper Article Sentences (JNAS) database [14]. Each pre-stored target speaker uttered 50 phoneme-balanced sentences included in one of seven sub-sets as shown in Table 1 . The source male speaker was not included in JNAS and uttered all of the seven sub-sets and an additional sub-set used for evaluation. We prepared parallel data sets between the source and each pre-stored target speaker by performing DTW automatically.

In evaluation, we used 10 target speakers consisting of five male and five female speakers not included in the pre-stored target speakers. We used 1 to 32 utterances for adapting the EV-GMM, and 21 utterances for evaluation. In the first E-step to estimate the adaptive parameters, i.e., the weight vector, we used TI-GMM as an initial model.

We used 24-dimensional mel-cepstrum as a spectral feature, which was extracted from smoothed spectrum analyzed by STRAIGHT [15]. We trained several EV-GMMs while changing the number of representative vectors as shown in Table 2. The number of mixture components was 128.

We converted source fundamental frequency $F_{0}$ to that of the target as follows:

$$
\log \tilde{F}_{0}=\frac{\sigma^{(y)}}{\sigma^{(x)}}\left(\log F_{0}-\mu^{(x)}\right)+\mu^{(y)},
$$

where $\mu^{(x)}$ and $\sigma^{(x)}$ denote mean and standard deviation of $\log$-scaled source $F_{0}$, and $\mu^{(y)}$ and $\sigma^{(y)}$ denote those of logscaled target $F_{0}$. The source speaker's parameters were cal- 


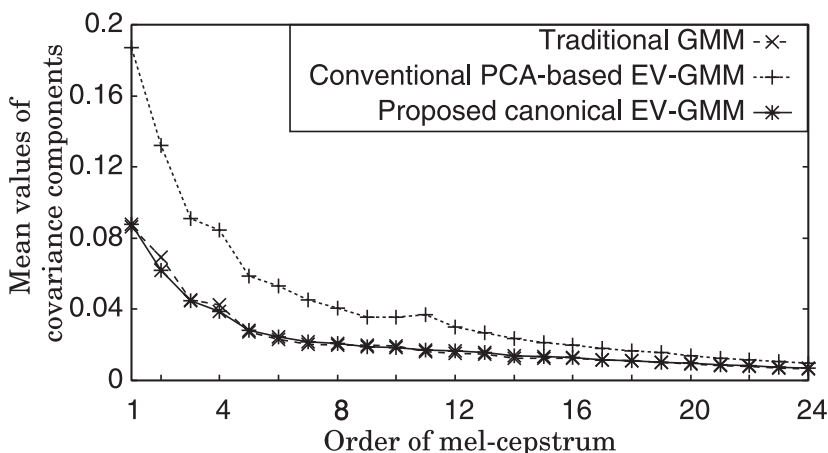

Fig. 3 Mean values of target covariance components of individual GMMs.

culated from the training data and the target speakers' parameters were calculated from the adaptation data.

\subsection{Objective Evaluations}

To investigate the effectiveness of the proposed adaptive training for alleviating the mismatches of probability density as mentioned in Sect. 2.5, we compared static feature components of the target covariance matrices $\boldsymbol{\Sigma}_{m}^{(Y Y)}$ of the conventional PCA-based EV-GMM with those of the proposed canonical EV-GMM. In the proposed adaptive training, we set the number of representative vectors to 159 and used the TI-GMM as an initial model. Figure 3 shows mean values of those covariance components over all mixture components. It also shows those values averaged over traditional GMMs separately trained using individual parallel data sets of the source and the target speakers. The covariance values of the PCA-based EV-GMM are larger than those of the traditional GMMs because the PCA-based EV-GMM models acoustic variations among many pre-stored target speakers. On the other hand, the covariance values of the canonical EV-GMM are almost equal to those of the traditional GMMs. This result shows that the proposed adaptive training is capable of effectively reducing the influence of the inter-speaker variations on the EV-GMM training.

To demonstrate the effectiveness of the proposed adaptive training in spectral conversion accuracy, we evaluated mel-cepstral distortion between the target and converted features when using the conventional PCA-based EV-GMM and the proposed canonical EV-GMM. These EV-GMMs were the same as those evaluated in Fig. 3. Figure 4 shows mel-cepstral distortion as a function of the number of adaptation utterances. We can see that the proposed canonical EV-GMM always outperforms the conventional PCA-based EV-GMM. Therefore, the proposed adaptive training is effective for improving spectral conversion accuracy in oneto-many EVC.

To investigate the influence of a local optimum problem as described in Sect.3.2, we evaluated spectral conversion accuracy using the four EV-GMMs: 1) PCA-based EV-GMM "Conventional"; 2) the canonical EV-GMM "Proposed (TI-GMM)" trained using the TI-GMM as an initial

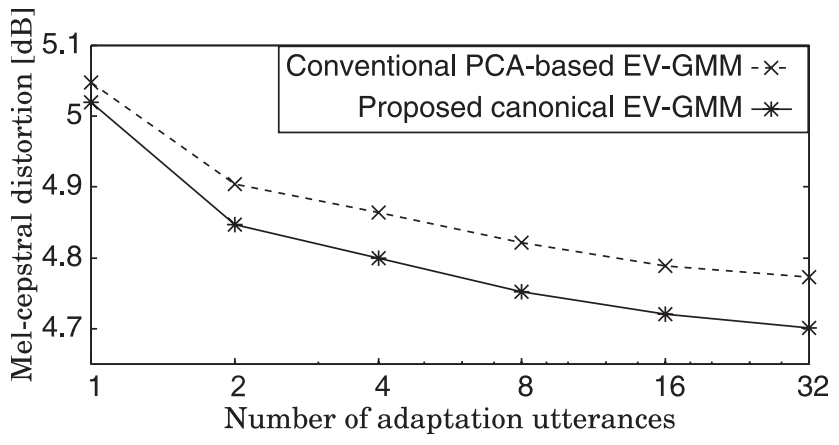

Fig. 4 Mel-cepstral distortion as a function of the number of adaptation utterances.

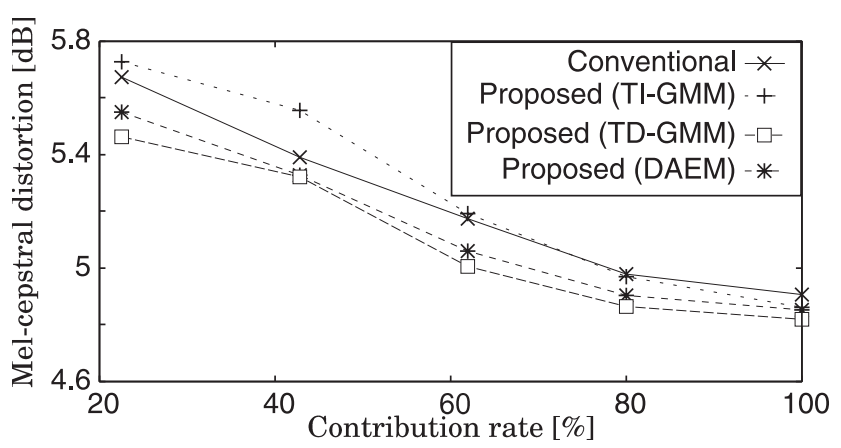

Fig. 5 Mel-cepstral distortion as a function of the contribution rate. The number of adaptation utterances is set to two.

model; 3) the canonical EV-GMM "Proposed (TD-GMM)" trained with the occupancy approximation as mentioned in Sect.3.2.1; and 4) the canonical EV-GMM "Proposed (DAEM)" trained with DAEM algorithm. Figure 5 shows mel-cepstral distortion as a function of the contribution rates of the representative vectors as shown in Table 2. Although the "Proposed (TI-GMM)" method yields performance improvement when the contribution rate is $100 \%$, it causes performance degradation when the contribution rate is less than $60 \%$. If using a large enough number of the representative vectors so that acoustic characteristics of individual pre-stored target speakers are modeled well, the EV-GMM training doesn't cause a severe local optimum problem. On the other hand, if the number of representative vectors is too small to span a subspace modeling those acoustic characteristics precisely, the EV-GMM training significantly suffers from a local optimum problem. This problem is effectively addressed by introducing the occupancy approximation "Proposed (TD-GMM)" or the DAEM algorithm "Proposed (DAEM)" to the EV-GMM training. It is interesting that the occupancy approximation outperforms the DAEM algorithm in particular when the contribution rate is $20 \%$. It would be expected that the mixture-component occupancies calculated by the TD-GMM would give better ML estimates of model parameters since they are quite similar to those calculated by reasonable model parameters such as the canonical EV-GMM with 159 representative vectors as shown in Fig. 2. Although the occupancy approximation is a heuristic 
approach not supported mathematically, it is more computationally efficient than the DAEM algorithm. Therefore, it is a very effective approach for alleviating the local optimum problem.

\subsection{Subjective Evaluations}

To demonstrate the effectiveness of the proposed method, we conducted a preference test on speech quality and an $\mathrm{XAB}$ test on conversion accuracy for speaker individuality. In these tests, the proposed canonical EV-GMM evaluated in Fig. 3 was compared with the conventional PCA-based EVGMM. In the preference test, a pair of two different types of the converted speech was presented to listeners, and then they were asked which voice sounded better. In the XAB test, a pair of two different types of the converted speech was presented to them after presenting the target speech as a reference. Then, they were asked which voice sounded more similar to the reference target. The number of listeners was five. Three utterances were randomly selected from 21 test utterances to make up a test set for each listener, and each utterance was converted into 10 target speakers' voices, leading to 30 sample-pairs. Each listener evaluated 60 sample-pairs consisting of those 30 sample-pairs and another 30 sample-pairs generated by swapping the order of presenting samples in each pair. Totally, 15 different utterances were used in these tests. The number of adaptation utterances was set to two in each evaluation

Figure 6 shows the results of each subjective evaluation. We can see that 1) the proposed method yields significant improvement in speech quality and 2) conversion accuracy for speaker individuality by the proposed method is almost equal to that by the conventional method. These results suggest that the proposed adaptive training is very effective for improving the performance of one-to-many EVC.

We also conducted another preference test on speech quality to evaluate the effectiveness of the proposed methods for alleviating the local optimum problem. The four EVGMMs evaluated in Fig. 5 were compared with each other. In this test, the number of representative vectors was set to one and the number of adaptation sentences was set to two. The number of listeners was 10 . To make up a test set for each listener, four utterances were randomly selected from 21 test utterances and two target speakers were also randomly selected from 10 target speakers. Two of the four utterances were converted so that they were uttered by one of the two target speakers and the remaining two utterances were converted so that they were uttered by the other target speaker. Six sample-pairs were generated with four types of the converted speech for each utterance, leading to 24 sample-pairs. Each listener evaluated 48 sample-pairs consisting of those 24 sample-pairs and another 24 sample-pairs generated by swapping the presentation order of the samples in each pair.

Figure 7 shows the result of the preference test on speech quality. "Proposed (TI-GMM)" causes performance degradation because of a local optimum problem. On the

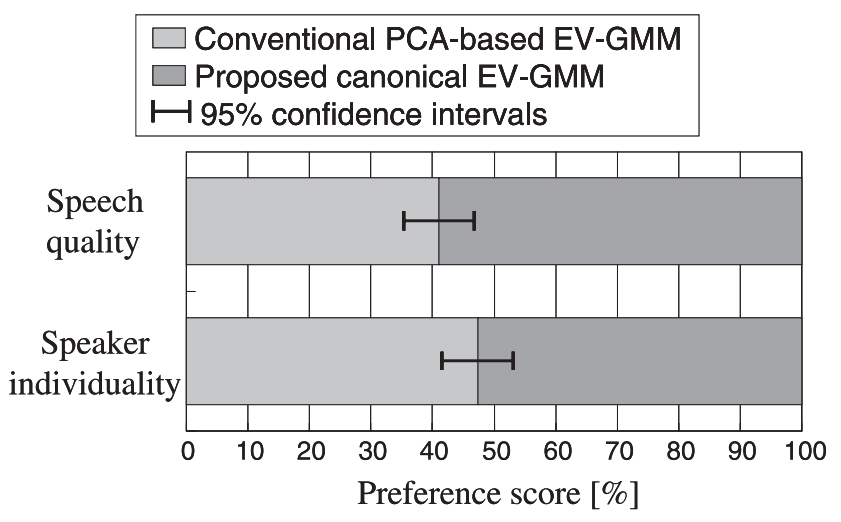

Fig. 6 Results of subjective evaluation.

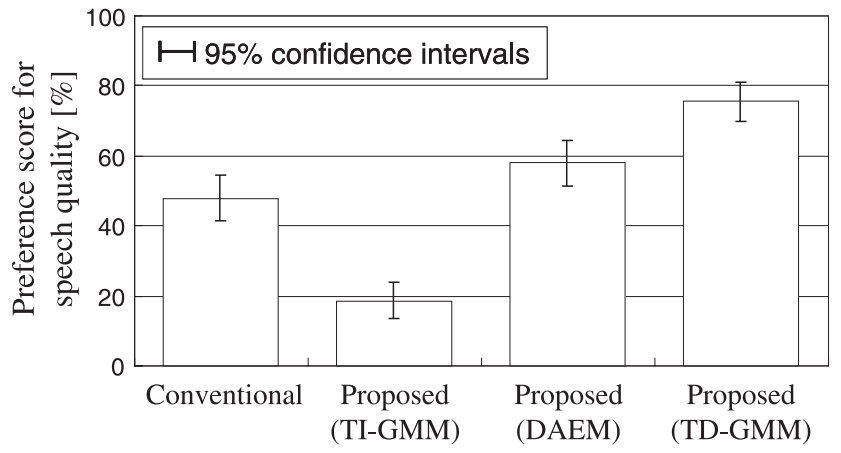

Fig. 7 Result of preference test on speech quality when the contribution rate is set to $20 \%$.

other hand, the local optimum problem is effectively alleviated by "Proposed (TD-GMM)" or "Proposed (DAEM)". These results are consistent with those observed in Fig. 5.

\section{Conclusion}

In order to improve the performance of one-to-many eigenvoice conversion (EVC), we have proposed an adaptive training method for the eigenvoice Gaussian mixture model (EV-GMM). The conventional EV-GMM parameters were affected by inter-speaker acoustic variations because they were determined based on a target-speaker-independent GMM (TI-GMM). These parameters often caused the degradation of the conversion performance. To address this problem, we have applied the proposed adaptive training to the EV-GMM. Moreover, we have also proposed a few approaches to alleviating the local optimum problem observed in the EV-GMM training. We have evaluated the effectiveness of the proposed methods objectively and subjectively. The experimental results have demonstrated that the proposed training method is very effective for improving the performance of the one-to-many EVC.

\section{Acknowledgment}

This work was supported in part by MIC SCOPE. 


\section{References}

[1] M. Abe, S. Nakamura, K. Shikano, and H. Kuwabara, "Voice conversion through vector quantization,” J. Acoust. Soc. Jpn. (E), vol.1, no.2, pp.71-76, 1990.

[2] M. Abe, K. Shikano, and H. Kuwabara, "Statistical analysis of bilingual speaker's speech for cross-language voice conversion," J. Acoust. Soc. Am., vol.90, no.1, pp.76-82, 1991.

[3] M. Mashimo, T. Toda, Kawanami, K. Shikano, and N. Campbell, "Cross-language voice conversion evaluation using bilingual databases," IPSJ Journal, vol.43, no.7, pp.2177-2185, 2002.

[4] Y. Stylianou, O. Cappé, and E. Moulines, "Continuous probabilistic transform for voice conversion," IEEE Trans. Speech Audio Process., vol.6, no.2, pp.131-142, 1998.

[5] T. Toda, A.W. Black, and K. Tokuda, "Voice conversion based on maximum likelihood estimation of spectral parameter trajectory," IEEE Trans. Audio, Speech and Language Processing, vol.15, no.8, pp.2222-2235, Nov. 2007.

[6] A. Mouchtaris, J. der Spiegel, and P. Mueller, "Non-parallel training for voice conversion based on a parameter adaptation approach,' IEEE Trans. Audio, Speech and Language Processing, vol.14, no.3, pp.952-963, May 2006.

[7] C.H. Lee and C.H. Wu, "Map-based adaptation for speech conversion using adaptation data selection and non-parallel training," Proc. Interspeech 2006 - ICSLP, pp.2254-2257, Sept. 2006.

[8] T. Toda, Y. Ohtani, and K. Shikano, "Eigenvoice conversion based on Gaussian mixture model," Proc. Interspeech 2006 - ICSLP, pp.2446-2449, Sept. 2006.

[9] R. Kuhn, J. Junqua, P. Nguyen, and N. Niedzielski, "Rapid speaker adaptation in eigenvoice space," IEEE Trans. Speech Audio Process, vol.8, no.6, pp.695-707, April 2000.

[10] T. Toda, Y. Ohtani, and K. Shikano, "One-to-many and many-toone voice conversion based on eigenvoices," Proc. ICASSP, vol.4, pp.1249-1252, April 2007.

[11] T. Anastasakos, J. McDonough, R. Schwartz, and J. Makhoul, "A compact model for speaker-adaptive training," Proc. ICSLP, vol.2, pp.1137-1140, 1996

[12] M.J.F. Gales, "Cluster adaptive training for hidden Markov models," IEEE Trans. Speech Audio Process. vol.8, no.4, pp.417-428, 2000.

[13] N. Ueda and R. Nakano, "Deterministic annealing EM algorithm," IEICE Trans. Inf. \& Syst. (Japanese Edition), vol.J80-D-II, no.1, pp.267-276, Jan. 1997

[14] “Jnas: Japanese newspaper article sentences,' http://www.milab.is.tsukuba.ac.jp/jnas/instruct.html

[15] H. Kawahara, I. Masuda-Katsuse, and A. de Cheveigné, "Restructuring speech representations using a pitch-adaptive time-frequency smoothing and instantaneous frequency-based fo extraction: Possible role of a repetitive structure in sounds," Speech Commun., vol.27, no.3-4, pp.187-207, 1999.

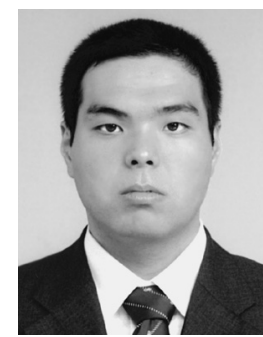

Yamato Ohtani was born in Osaka, Japan on September 8, 1980. He received the B.E. degree in engineering from Osaka University, Osaka, Japan, in 2005 and the M.E. degree in engineering from the Graduate School of Information Science, Nara Institute of Science and Technology (NAIST), Nara, Japan, in 2007. He is now Ph.D. student in NAIST. He was an intern researcher at ATR Spoken Language Communication Research Laboratories (ATR-SLC) from 2006 to 2009 . He is a student member of ISCA

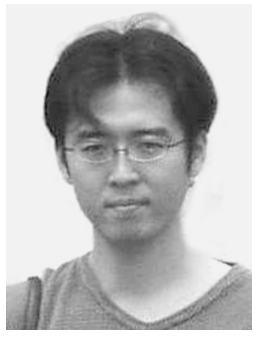

Tomoki Toda was born in Aichi, Japan on January 18, 1977. He received the B.E. degree in electrical engineering from Nagoya University, Nagoya, Japan, in 1999 and the M.E. and D.E. degrees in engineering from the Graduate School of Information Science, Nara Institute of Science and Technology (NAIST), Nara, Japan, in 2001 and 2003, respectively. He was a Research Fellow of the JSPS in the Graduate School of Engineering, Nagoya Institute of Technology from 2003 to 2005 . He is currently an Assistant Professor of the Graduate School of Information Science, NAIST. From March 2001 to March 2003, he was an Intern Researcher at the ATR Spoken Language Translation Research Laboratories, Kyoto, Japan, and then he was a Visiting Researcher at the ATR until March 2006. $\mathrm{He}$ has been a Visiting Researcher at the NICT, Kyoto, Japan, since May 2006. He was also a Visiting Researcher at the Language Technologies Institute, CMU, Pittsburgh, USA, from October 2003 to September 2004 and at the Department of Engineering, University of Cambridge, Cambridge, UK, from March 2008 to August 2008, respectively. His research interests include statistical approaches to speech processing such as voice transformation, speech synthesis, speech analysis, speech production, and speech recognition. He received the TELECOM System Technology Award for Students and the TELECOM System Technology Award from the TAF, in 2003 and 2008, respectively. He also received the ISS Best Paper Award from the IEICE, Japan, in 2008, the Ericsson Young Scientist Award from Nippon Ericsson K. K. in 2008, and both the Awaya Prize Young Researcher Award and the Itakura Prize Innovative Young Researcher Award from the ASJ in 2009. He has been a member of the Speech and Language Technical Committee of the IEEE SPS since January 2007. He is a member of IEEE, ISCA, IPSJ, and ASJ.

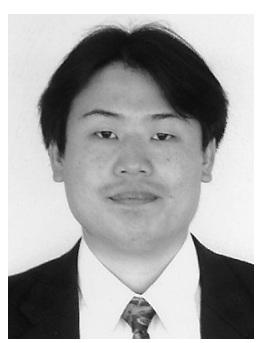

Hiroshi Saruwatari was born in Nagoya, Japan, on July 27, 1967. He received the B.E., M.E. and Ph.D. degrees in electrical engineering from Nagoya University, Nagoya, Japan, in 1991, 1993 and 2000, respectively. He joined Intelligent Systems Laboratory, SECOM CO., LTD., Mitaka, Tokyo, Japan, in 1993, where he engaged in the research and development on the ultrasonic array system for the acoustic imaging. $\mathrm{He}$ is currently an associate professor of Graduate School of Information Science, Nara Institute of Science and Technology. His research interests include array signal processing, blind source separation, and sound field reproduction. He received the Paper Awards from IEICE in 2001 and 2006, and from TAF in 2003. He is a member of the IEEE and the Acoustical Society of Japan. 


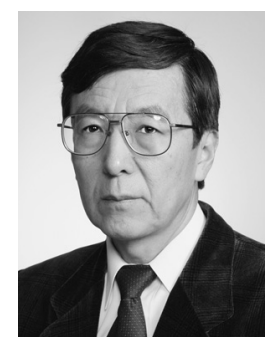

Kiyohiro Shikano received the B.S., M.S., and $\mathrm{Ph} . \mathrm{D}$. degrees in electrical engineering from Nagoya University in 1970, 1972, and 1980, respectively. He is currently a professor of Nara Institute of Science and Technology (NAIST), where he is directing speech and acoustics laboratory. From 1972 to 1993 , he had been working at NTT Laboratories. During 1986-1990, he was the Head of Speech Processing Department at ATR Interpreting Telephony Research Laboratories. During 1984-1986, he was a visiting scientist in Carnegie Mellon University. He received the Yonezawa Prize from IEICE in 1975, the Signal Processing Society 1990 Senior Award from IEEE in 1991, the Technical Development Award from ASJ in 1994, IPSJ Yamashita SIG Research Award in 2000, and Paper Award from the Virtual Reality Society of Japan in 2001, IEICE paper award in 2005 and 2006, and Inose award in 2005. He is a fellow of the Institute of Electrical and Electronics, Engineers (IEEE), and Information Processing Society of Japan, and a member of the Acoustical Society of Japan (ASJ) and International Speech Communication Society (ISCA). 